\title{
Data Collection Method for Mobile Control Sink Node in Wireless Sensor Network Based on Compressive Sensing
}

\author{
Yongfa Ling $^{1}$, Hui Wang ${ }^{1}$ \\ ${ }^{1}$ Hezhou University, School of Mechanical and Electronic Engineering, 542899 Hezhou, China
}

\begin{abstract}
The paper proposes a mobile control sink node data collection method in the wireless sensor network based on compressive sensing. This method, with regular track, selects the optimal data collection points in the monitoring area via the disc method, calcu-lates the shortest path by using the quantum genetic algorithm, and hence determines the data collection route. Simulation results show that this method has higher network throughput and better energy efficiency, capable of collecting a huge amount of data with balanced energy consumption in the network.

Keywords: Wireless Sensor Network. Compressive Sensing. Mobile Control Node. Quantum Genetic Algorithm
\end{abstract}

\section{Introduction}

Wireless sensor network uses a huge number of randomly deployed nodes to cover the monitoring area, forms a multi-hop ad hoc network system through wireless communication, and thus performs long-term automatic monitoring, collection and transmission of environmental data. It is a newly-developed technology integrated with and penetrated by embedded systems, wireless communications technologies, network technologies, and micro-electromechanical systems etc.; a low-cost, lowpower autonomous network system consisting of tiny sensor nodes with sensing, computing and communication capacities; and a "smart" system able to independently perform various monitoring tasks according to the environment[1]. Wireless sensor network has a wide range of uses, among which data collection is one of its fundamental applications. The traditional approach of data collection is to fixate the positions of all nodes, collect data and then submit the data to the sink node with the aid of routing protocol. Its main problems include: (1) connectivity constraint, which indicates that since the network is not fully connected, it is inevitable that data collected from some nodes can not be submitted; (2) funnel effect, which indicates that nodes closer to the sink node will consume more energy, network partition will occur earlier, so do operation with huge data[2].

The compressive sensing theory offers a new data acquisition mode for wireless sensor networks [3]. According to the compressive sensing theory, a sparse signal can be accurately reconstructed with a small number of samples, and the sampling can be completed by linear projection of test data, which allows sensor nodes to acquire data in a compressive manner, without any need of additional computational overhead. Despite of many good features such as easy construction, strong adaptability, and high transmission efficiency, wireless sensor networks are also troubled by some constraints in terms of energy supply, sensor life cycle, time delay, bandwidth, signal distortion, and transmission expense and so on. Moreover, nodes in the wireless sensor network also need separate energy supply. Therefore, energy consumption is an important factor deciding the life cycle of sensor nodes. The integration of compressive sensing theory and wireless sensor network offers an effective solution to these problems [4], which optimizes the energy consumption of sensor nodes [5], and allows sparse signals in the wireless sensor network to be accurately reconstructed even with few sampling times [6]. In essence, such an integration provides an optimal computation method based on mathematical upper limit, for the acquisition of sparse information. Because of its openness, wireless sensor network is vulnerable to the influence of environmental noise, especially when using compressive sensing to make under-sampling. Although the network can reduce the expenditure for data acquisition, such "incomplete" under-sampling data is more sensitive to noise [7].

The above shortcomings are partially rooted in the stationary feature of the sink node. After the proposals of RacemoteZ, quadcopter and other mobile nodes, some scholars put forward acquisition schemes based on mobile nodes, which partially solved the aforementioned problems and brought about advantages in network throughout, transmission reliability and other aspects. The problem of these methods lies in that it is difficult to determine the moving route of the sink node and there exists a certain degree of time delay. Most data acquisition applications have less demanding requirements on real-time performance, controlling the movement of sink node becomes the key. Some research 
findings have been published in this aspect, and classical strategies include random movement, movement along the network edge, movement toward the node with the most sending data, and movement toward the node with the most remaining energy etc. Some of these methods fail to consider the distribution of nodes, and some cost large computational overhead, none of which is suitable for wireless sensor network and compressive sensing.

\section{Related Models and Assumptions}

To sum up, this paper attempts to propose a Data Collection Method with Regular Track (hereinafter referred to as DCMR). The moving strategy of DCMR includes two steps. The first step is to select an array of collection points according to the distribution of nodes, while the second step is to identify the shortest loop circuit passing these points. This circuit will then serve as the data collection route of the sink node that moves along the circuit to collect data. According to research of Akkaya et al. [8], the optimal collection point in the network is an NP issue, while the calculation of the shortest loop circuit is a traveling issue, which also can be classified as an NP issue. Since there are no extremely good processing methods for these two steps, this paper tries to make some simplification and approximation. When selecting collection points, DCMR tries to select as few collection points as possible and uses the quantum genetic algorithm for the calculation of the shortest loop circuit [9].

The above scheme makes the following assumptions for the wireless sensor network based on compressive sensing. (1) Sensor node. All sensor nodes in the network are of the same type and with the same initial energy, each equipped with some storage space. (2) Monitoring scene. The target area is a plane domain, so the monitoring area is relatively flat and absent of obstacles. (3) Sink node. The sink node has plenty of battery power, relatively high storage capacity and some degree of moving ability. The communication distance of the sink node is identical to that of ordinary nodes. (4) Node deployment. Nodes are randomly deployed and become unmovable after deployment. (5) Data submission mode. Passive submission. The node saves acquired data into its cache, and submits the data only upon notice from the sink node. (6) Operation Type. Periodic collection. The node periodically collects data from the sensor. Data collection operation has no special demand on transmission delay.

Among them, the sink node is a key step, which realizes mobility with the aid of RacemoteZ, quadcopter and other mobile nodes and ensures energy supply with large-capacity batteries or regular battery charging. In addition, when calculating the moving route of the sink node, this paper does not consider the constraints of road conditions on the moving track, which means that the sink node can move freely. Surely, if the sink node is the quadcopter and other flight sensor nodes, this problem will no longer exist.

\section{General Idea and Procedures}

\subsection{General Idea}

The DCMR scheme uses mobile sink nodes to collect perception data generated in the network. Mobile paths have a greater impact on the acquisition performance in the network. In DCMR protocol, the moving path of sink node is determined by the following two steps: (1) determine the collection point: first, determine the position of every disc and select a collection point in the disc; (2) calculate the short-est path: utilize the quantum genetic algorithm to identify the shortest loop circuit through these points.

In the first step, DCMR protocol needs to select out an array of acquisition points from the monitoring area. How to determine the optimal collection points in the network is an NP issue, for which there are no good processing methods, so our goal is to handle as few collection points as possible in the network, which can not only help reduce the calculation amount in the second step but also work out the shortest path. In the wireless sensor network, the communication range of sink node around every collection point can be regarded as a disc centered around that collection point. Therefore, the mission in the first step is to find a set of coordi-nate points satisfying the following two conditions: (1) as few coordinate points as possible; (2) a collection of r-radius discs centering on these coordinate points able to cover the monitoring area. In the second step, DCMR calculates the shortest loop circuit passing these collection points, which not only reduces the energy consumption for the move-ment of the sink node but also cuts down the time delay of data submission. Since the shortest loop circuit is also an NP issue, DCMR chooses the quantum genetic algorithm for calculation. Based on the traditional genetic algorithm, the quantum genetic algorithm brings in the concept of quantum calculation, hugely improving its convergence rate and solution-finding ability.

\subsection{Procedures}

The procedures of data collection are illustrated as follows. (1) Initialization. All nodes report their positions to the sink node; (2) Path calculation. According to the location of all nodes, the sink node finds out all acquisition points in the network and works out the shortest loop circuit passing these points; (3) Data collection. The sink node moves along the calculated route, and broadcasts a collection notice once reaching a collection point. Nearby nodes will submit the saved data upon receiving the notice. To reduce channel conflicts, every node should wait for a random spell of time before submitting data.

During data collection, some nodes may perish after the network has been operat-ing for a certain period, so DCMR can restart calculating the moving route of sink node to adapt to the node distribution in the network. Preset trigger conditions in-clude timer, death toll and so on.

\section{Simulation Experiments and Conclusion}




\subsection{Test Scenarios and Indicators}

To validate the performance of DCMR, this paper conducts a comparison test among DCMR, Stationary Sink Node Mode, Random Roam Strategy, and Peripheral Protocol in the NS 2.34 environment. Among these four strategies, DCMR and Random adopts the passive submission mode, in which nodes store acquired data in the local machine and submit them to the sink node after receiving a notice. However, Stationary and Peripheral approaches take the active submission mode, in which nodes take the initiative to send collected data to the sink node with the aid of routing protocol. The setting of other experimental parameters is shown in Table 1.

Table 1 Setting of Experimental Parameters

\begin{tabular}{|l|l|}
\hline Parameter & Value \\
\hline Scene size & $100 * 100(\mathrm{~m} 2)$ \\
\hline Number of Nodes & $100,200,300,400$ \\
\hline Routing protocol & $\begin{array}{l}\text { Stationary \& Peripheral: } \\
\text { AODV } \\
\text { DCMR \& Random: None }\end{array}$ \\
\hline MAC protocol & 802.11 \\
\hline Node initial energy & $1.8 \mathrm{~J}$ \\
\hline Data acquisition speed & $40 \mathrm{~KB} / 10 \mathrm{~s}$ \\
\hline $\begin{array}{l}\text { Node communication } \\
\text { radius }\end{array}$ & $10 \mathrm{~m}$ \\
\hline Moving speed of sink node & $\begin{array}{l}\text { Stationary: } 0(\mathrm{~m} / \mathrm{s}) \\
\text { Other protocols: } 5,25(\mathrm{~m} / \mathrm{s})\end{array}$ \\
\hline
\end{tabular}

During the experimental test, this paper mainly examines the following six in-dicators. (1) Throughput: The ratio of the number of all data packets in the net-work to the working time of the network. Data packets in the network include sent data packets, resent data packets due to conflicts, data packets forwarded by nodes, routing query packets and routing control packets etc. (2) The amount of data collected: It refers to the number of valid data packets that have reached the sink node. The so-called valid data packets refer to packets containing ac-quired data. (3) Remaining energy: It refers to the remaining energy of all nodes in the network when the network fails to work properly, also at a time when the sink node collects no data. (4) Efficiency: It refers to the ratio of the total number of valid data packets received by the sink node to the total number of all data pack-ets in the network. (5) Average delay: It refers to the mean value of time delay of all valid data packets that have reached the sink node. (6) Energy efficiency rate: It refers to the ratio of the energy consumption of data packets received by the sink node to the total energy consumption in the entire network.

\subsection{Conclusion}

Experimental results show that DCMR method has better performance in terms of network throughput, energy efficiency and other aspects, and collects more da-ta.

The use of mobile sink node can improve network throughput, balance the energy consumption of nodes, enhance the efficiency of data acquisition, and work normally even in case of network partition. The DCMR method proposed in this paper shows all these advantages. Due to the limit of the moving speed of sink node, there will be some degree of time delay in data collection. Therefore, DCMR method has promising application prospects for data collection tasks that contain a huge amount of data and have no demanding requirements on time limit.

If we can introduce data fusion, node scheduling, parallel transmission and other technologies to sensor nodes and integrate them with DCMR method, net-work performance will be further improved.

\section{ACKNOWLEDGMENT}

This research was supported by the doctor's scientific research foundation of Hezhou University

\section{References}

1. Stankovic J A, Abdelzaher F, Lu C Y, Sha L and Hou J C. Real-Time Communication and Coordination in Embedded Sensor Networks[C] //Proceedings of the IEEE, 2003, 91(7): 1002-1022.

2. Jian Guo. Research on Several Key Technologies of Data Processing in Wireless Sensor Networks[D]. Nanjing University of Posts and Telecommunications, 2012:39-40.

3. 3. Chen Shan-Xiong, Xiong Hai-Ling, Peng Xi-Hua, $\mathrm{Wu}$ Sheng. Intrusion detection based on compressed sensing. ICIC Express Letters, 2013, 7(10): 31693176.

4. 4. Aeron S, Saligrama V, Zhao Manqi. Information theoretic bounds for compressed sensing. IEEE Transactions on Information Theory, 2010, 56(10): 5111-5130.

5. 5. Zhuang Xiaoyan, Wang HOujun, Dai Zhijian. Wireless sensor networks based on compressed sensing//Proceedings of the 2010 3rd IEEE International Conference on Computer Science and Information Technology(ICCSIT). Chendu, China, 2010: 90-92.

6. 6. Pepe A, Rodriguez M A. Collaboration in sensor network research: An in-depth longitudinal analysis of assortative mixing patterns. Scientometrics, 2010, 84(3): 687-701.

7. 7. Chen Shanxiong, He Zhongshi, Xiong Hailing, Liao Jianwei. A Reconstruction Algorithm of Wireless Sensor Signal Based on Compressed Sensing[J],Chinese Journal of Comput-ers,2015, 38(3):614-622.

8. 8. Akkaya K, Younis M, Bangad M. Sink repositioning for enhanced performance in wireless sensor networks [J]. Computer Networks, 2005, 49(4): 512-534.

9. 9. Yang J A, Zhuang Z Q. Research of Quantum Genetic Algorithm and Its Application in Blind Source Separation [J]. Journal of Electronics, 2003, 20(1): 62-68. 